\title{
Generalized Entropy and Temperature in Nuclear Multifragmentation
}

\author{
A. Atalmi, M. Baldo, G. F. Burgio and A. Rapisarda \\ Istituto Nazionale di Fisica Nucleare, Sezione di Catania and Dipartimento di Fisica, \\ Universitá di Catania, Corso Italia 57, I-95129 Catania, Italy
}

(November 5, 2018)

\begin{abstract}
In the framework of a 2D Vlasov model, we study the time evolution of the "coarse-grained" Generalized Entropy (GE) in a nuclear system which undergoes a multifragmentation (MF) phase transition. We investigate the GE both for the gas and the fragments (surface and bulk part respectively). We find that the formation of the surface causes the growth of the GE during the process of fragmentation. This quantity then characterizes the MF and confirms the crucial role of deterministic chaos in filling the new available phase-space: at variance with the exact time evolution, no entropy change is found when the linear response is applied. Numerical simulations were used also to extract information about final temperatures of the fragments. From a fitting of the momentum distribution with a Fermi-Dirac function we extract the temperature of the fragments at the end of the process. We calculate also the gas temperature by averaging over the available phase space. The latter is a few times larger than the former, indicating a gas not in equilibrium. Though the model is very schematic, this fact seems to be very general and could explain the discrepancy found in experimental data when using the slope of light particles spectra instead of the double ratio of isotope yields
\end{abstract}


method in order to extract the nuclear caloric curve.

\section{PACS numbers: 25.70.Pq, 24.60.Lz,21.65.+f}

Multifragmentation phase transition is one of the most interesting recent discoveries in nuclear physics [1, [7]. In the last years an intense effort has been put forward both experimentally [3 9] and theoretically 92 23] in order to understand this phenomenon. Several scenarios have been proposed for the onset mechanism and for the dynamics underlying the Nuclear Multifragmentation (MF). In the statistical model [10,11] one assumes that the energetically available phase space dominates the reaction dynamics. This implies that the set of multifragmentation events fills, all together, the phase space in an almost uniform fashion and therefore a quasi-static statistical description is possible. The model appears quite successful in describing some of the phenomenological features observed in many MF experiments, including the multiplicity and mass distributions at different asymmetries, as well as the more recently discussed "caloric curve", which some groups claim to have extracted from the observational data [3 6]. The model, however, does not provide a mechanism for the formation of the fragments along the dynamical evolution of the reaction, and for the filling of the available phase space (once the whole ensemble of multifragmentation events is considered). The latter feature implies a large (maximal) production of entropy, which is indeed proportional to the logarithm of the phase space volume occupied by the considered ensemble of events [11. A natural source of entropy is the process of collisions between particles, as, for instance, in the BUU model of nuclear reactions [12 14] or in molecular dynamics simulations (see for example ref. [17]). It has to be kept in mind, however, that if the system is chaotic [19 21,23], large fluctuations from one event to another must be expected, with a possible filling of the phase space by the ensemble of events, independently from the details of the microscopic mechanism.

In order to contribute to the clarification of the previous points, we discuss in the present paper new calculations of nuclear multifragmentation in a schematic and simplified 2D Vlasov model already well known in the literature [18 21. We start the system inside 
the mechanically unstable spinodal region and follow the birth and growth of fragments induced by a very small initial random noise. The latter simulates the missing initial dynamics and drives the system outside the spinodal region. In order to characterize this process we use the "coarse-grained" Generalized Entropy (GE) which is the non-equilibrium extension of the thermodynamical entropy [24]. GE has already been used in the past both theoretically [14] and experimentally [8] in order to investigate nuclear MF. Usually the increase of GE is believed to be due to two-body collisions. We find that GE grows even in a Vlasov approach if initialized inside the spinodal region. More precisely we find that GE grows as the surface of fragments is formed. This growth reflects the crucial role of chaoticity in MF confirming what has already been found in previous studies 19 21,23. Chaotic motion is the main mechanism responsible for dynamical filling of the available equilibrium phase space. This result seems to be very general in phase transitions as recently confirmed in several investigations 23,25,28].

Recently a very interesting link between the GE and the Kolmogorov-Sinai entropy (KS entropy) (the sum of the positive Lyapunov exponents) [29,30 has been found for chaotic maps [31]. Our results are consistent with this finding in that no entropy growth is observed when the linear response is applied (regular evolution) [21,22].

In the present paper we extract also information about the temperature of the fragments. We find also a gas of particles which is not in equilibrium with the fragments. The gas has a temperature which is a factor of 2-3 greater than the fragments' one. These non-equilibrated particles in a realistic situations will likely be emitted in a first stage of the process and do not carry information on the equilibrated component of the system which undergoes MF [5]. Though the model is very schematic this finding can be very general and explain the puzzling discrepancy observed experimentally between the caloric curve extracted from isotope yields [32] and the one from the slopes of light particle spectra [5,6].

This paper is organized as follows. In section I we remind the reader the details of the model. In section II we define the "coarse-grained" GE and discuss its connection to chaos and thermodynamical entropy. Numerical results are presented in section III and 
conclusions are drawn in section IV. We report in the appendix the method we used to extract the temperature.

\section{THE MODEL}

Our theoretical framework is based on the solution of the Vlasov equation for the onebody density $f$ in phase space

$$
\frac{\partial f(\mathbf{r}, \mathbf{p}, t)}{\partial t}=\{h[f], f\}
$$

Here $\{.,$.$\} is the Poisson bracket, so that eq.(11) represents the collisionless propagation$ of $f$ in the self-consistent one-body field described by the effective single-particle hamiltonian $h(\mathbf{r}, \mathbf{p})=p^{2} / 2 m+U(\mathbf{r}, \mathbf{p})$, being $\mathrm{U}$ the self-consistent mean field. Over the past decade the Vlasov equation has been widely used in nuclear mean-field theory for describing many aspects of intermediate energy heavy ion collisions. In particular it has been extended by incorporating a Pauli blocked collision term leading to a Boltzmann-like dynamical description, denoted the BUU 1 model [12,[13]. In the present paper no collision term has been considered, since we have already seen [19,20] that it is not very important inside the spinodal region.

In the present work the Vlasov equation has been solved numerically in a two-dimensional lattice using the same code of ref. [18], as already done in refs. [19,20]. We have studied a fermion gas situated in a two-dimensional periodic box whose size is kept constant during the evolution. The box sidelengths are equal to $L_{x}=51 \mathrm{fm}$ and $L_{y}=15 \mathrm{fm}$. We divided the single particle phase space into several small cells. We employed in momentum space $51 \mathrm{x} 51$ small cells of size $\Delta p_{x}=\Delta p_{y}=40 \mathrm{MeV} / \mathrm{c}$, while in coordinate space $\Delta x=0.3333 \mathrm{fm}$ and $\Delta y=15 \mathrm{fm}$. The initial local momentum distribution was assumed to be the one of a

\footnotetext{
${ }^{1}$ Various names have been adopted in literature, i.e. Boltzmann-Uehling-Uhlenbeck, LandauVlasov or Vlasov-Uehling-Uhlenbeck equation.
} 
Fermi gas at a fixed temperature $T$. For the effective one-body field we employed a simplified Skyrme interaction $U[\rho]=A\left(\bar{\rho}(x) / \rho_{0}\right)+B\left(\bar{\rho}(x) / \rho_{0}\right)^{2}$. The saturation density in two dimensions $\rho_{0}=0.55 \mathrm{fm}^{-2}$ corresponds to the usual three-dimensional Fermi momentum of $P_{F}=260 \mathrm{MeV} / \mathrm{c}$. Furthermore $\bar{\rho}(x)$ is the local average of the density with respect to the transverse direction $\mathrm{y}$, smeared in the $x$-direction with a gaussian of width $\mu=0.61 \mathrm{fm}$, in order to give a finite range to the interaction. The parameters of the force $A$ and $B$ have been chosen in order to reproduce correctly the binding energy of nuclear matter at zero temperature, and this gives $A=-100.3 \mathrm{MeV}$ and $B=48 \mathrm{MeV}$. Then a complete dynamical evolution is performed by subdividing the total time in small time steps, each equal to $\Delta t=0.5 \mathrm{fm} / \mathrm{c}$. The algorithm is deterministic, i.e. no noise of the Langevin-type has been used, and the total energy is conserved with a good accuracy. For more details concerning the mean field propagation on the lattice, the reader is referred to ref. [18].

\section{THERMODYNAMICAL ENTROPY, GENERALIZED ENTROPY AND CHAOS}

In this paper we study the amount of entropy generated during the time of fragment

formation. For this purpose a few considerations are necessary in order to introduce the notations and some quantities which will be essential in the numerical analysis.

Let us start by summarizing some basic concepts of statistical mechanics. Consider a hamiltonian system with $n$ degrees of freedom. The thermodynamical entropy $S_{0}$ is obviously defined only at thermodynamical equilibrium and is directly related to the number $N$ of microstates which correspond to a given macroscopic state. If we put equal to 1 the volume occupied in phase space by each one of the microstates (e.g. we put $\hbar=1$ in the quantal case), $N$ coincides also with the available microscopic phase space $\Omega$ (the volume of a $2 n$ dimensional manifold in the classical case). Historically this connection was introduced by Boltzmann and provides the statistical basis of thermodynamics [24, 33, 34]. In general, $S_{0}$ is just proportional to the logarithm of the number $N$ of the possible microscopic states 
in the considered macroscopic conditions. The latter defines the "ensemble" of microscopic states which correspond to the given macroscopic thermodynamical state. Therefore, in the microcanonical ensemble the entropy is just the logarithm of the microscopic available phase space $\Omega(E)$ at a given total energy $E$

$$
S_{0}=\ln N(E) \equiv \ln \Omega(E)=-\ln P(E)
$$

where $P(E)=N(E)^{-1} \equiv \Omega^{-1}$ is the probability of each one of the microstates, which are assumed to have all the same a priori probability.

Once the entropy is given in the microcanonical ensemble, all the other thermodynamical quantities can be extracted, or other ensembles can be introduced by means of a suitable Legendre transform.

Let a real macroscopic system be prepared in a generic microstate. Then, it is possible to describe the system according to standard statistical thermodynamics only if it is at least ergodic, namely the system in its temporal evolution will sample all the microstates (or an overwhelming majority of them) and with the same frequency. Since the energy is conserved, the asymptotic temporal average of the frequency will then coincide indeed with $P(E)$. However, it is essential to recognize that, for a system of identical particles, sets of very large number of equivalent configurations exist, namely the configurations which differ only by the rearrangement of particles among equivalent microstates. Among these sets, the one which contains the largest number of configurations will be the dominant one, since it will be the one with the highest occurrence frequency and statistical weight. At equilibrium the system will move mainly within this set of configurations (at least for large systems, for which fluctuations can be neglected). For instance, for a system of weakly interacting particles the dominant set is nothing but the one characterized by the canonical (Boltzmann) single particle occupation numbers. Of course, the time of approach to equilibrium is in general strongly dependent on the initial conditions and on how fast the system explores the available phase space.

In the physical conditions considered here, we are however more interested to what 
extent a set of initial conditions, taken all together, explore the available phase space during the evolution of the system. It is in fact the whole set of MF events which has to be considered, since it is the ensemble over which the relevant physical quantities have to be calculated. It can be also useful to consider different sub-sets of MF events, in order to study the fluctuations which are present within the whole MF set of events. In any case, this procedure is equivalent to the introduction of a Gibbs ensemble, namely a set of copies of the system at the initial time with slightly different initial conditions. Correspondingly, the ability of the set of initial conditions to reach equilibrium is usually referred to as the "mixing" property [34]. Mixing means that a generic set of nearby initial states, occupying a small initial volume $\omega_{o}$ in phase space, away from equilibrium, will spread rapidly throughout the available phase space, eventually dominated by the equilibrium configurations. It can be proved that mixing implies ergodicity [29,30].

This spreading, therefore, describes the evolution towards equilibrium of the system when initially prepared in a generic state inside the volume $\omega_{0}$. If one wants to describe quantitatively the approach to equilibrium, one can introduce a generalized and time dependent entropy just as the logarithm of the volume $\omega(t)$ occupied at time $t$ by the states originating from the set of initial conditions inside the volume $\omega_{0}$ at $t=0$. In other words, one can follow the evolution of the initial volume $\omega_{0}$ under the automorphism defined by the equations of motion.

As it is well known [34], this approach faces a well defined difficulty in the case of hamiltonian systems : due to Liouville theorem, the volume in phase space is conserved, and according to the above mentioned definition no entropy could be produced.

However, the filling of phase space for a hamiltonian system which displays chaoticity can be very intricate and "filamentary". In this case a "coarse grained" description appears quite natural, and the introduction of a GE function is possible and useful [34]. We illustrate this point with an example, taken from the literature on dynamical systems [30. Let us consider the standard map, which has the property of conserving the phase space area, and therefore it is quite analogous to a hamiltonian system. Actually it can be recasted into a 
hamiltonian form [30]. If we consider a set of nearby initial conditions, confined inside the square depicted in fig.1 (top panels), the map

$$
\begin{array}{ll}
y_{n+1}=y_{n}+\frac{K}{2 \pi} \sin \left(2 \pi x_{n}\right) & \bmod 1 \\
x_{n+1}=x_{n}+y_{n+1} & \bmod 1
\end{array}
$$

( $\mathrm{K}$ is the coupling term) will spread the initial occupied volume into a very complex and scattered structure, as the number of iterations $n$ increases. We report the time evolution for two values of the coupling, i.e. $K=0.05$ and $K=2$. In the first case one has an almost regular evolution, while in the second case a chaotic dynamics sets in. The more chaotic is the map, the larger is the area of phase space where the ensemble of points is scattered. In the case $\mathrm{K}=2$, after only a few iterations the equilibrium phase space is filled almost uniformly by a net of fine structures. One can compare this mixing property of such a chaotic map with the diffusion of a drop of ink inside a glass of water. On the other hand the quasi-regular case $(\mathrm{K}=0.05)$ would correspond to a drop of mercury.

In order to study the filling of phase space one can then divide the available phase space into a set of "coarse grained" cells, and take the fraction $p_{i}$ of the filled area inside each cell $i$. Equivalently, if one considers a set of representative points inside the initial square, one can define $p_{i}$ as the fraction of points inside the cell $i$ at a given time $t$. The coarse grained GE can be then defined by

$$
S=-\sum_{i} p_{i} \ln p_{i}
$$

In a "fine grained" description, the cell size is considered arbitrarily small, thus one has $p_{i}=0$ or $p_{i}=1$, and the entropy is identically zero. At variance, in a coarse grained description, $0<p_{i}<1$, so that $S$ is different from zero and it increases as the phase space filling increases. If the spread is uniform in phase space, one obtains the standard thermodynamical equilibrium entropy, with the microstates specified by the $\mathrm{N}$ cells of the lattice. In fact, in this case $p_{i}=1 / N$ and $S=\ln N=S_{0}$. 
The increase of entropy in the considered example as a function of the number of iterations is reported in fig.1 (bottom panel) for the two cases. Please notice that this definition of GE can be introduced also for non-mixing systems, for which the available phase space is not necessarily full or uniformly filled in the large time limit. Therefore the GE is a very useful tool to measure how big is the available equilibrium phase space once a proper grid is given to measure it. In general the definition of GE is not unique and depends on the grid size 34.

It has to be stressed that this definition of GE is physically different from the one adopted in the theory of dynamical systems by Kolmogorov [29]. The KS entropy is the sum of the positive Lyapunov exponents [30]. Recently a very interesting connection between the GE and the KS entropy has been found for chaotic maps [31]: the latter corresponds to the slope of the linear growth rate of the GE. Though this result is not a theorem and at the moment it is not clear if it can be simply extrapolated to systems with many degrees of freedom as the one we have investigated, a growth of GE is a strong hint of chaoticity. In the following we will discuss numerical results which support this conjecture.

A coarse grained description is introduced in the Vlasov equation (11), once it is written for a discrete grid. Through the GE we can in fact investigate the filling of phase space in MF.

In this case the differential equation (1) becomes a set of finite difference equations

$$
\frac{\partial f(\mathbf{r}, \mathbf{p})}{\partial t}+\mathbf{v}(\mathbf{p}) \cdot \Delta^{r} f+\mathbf{F}(\mathbf{r}) \cdot \Delta^{p} f=0
$$

where (the index $i$ labels the vector components)

$$
\begin{aligned}
\Delta_{i}^{r} f & =\frac{f\left(\mathbf{r}+\mathbf{n}_{i}, \mathbf{p}\right)-f\left(\mathbf{r}-\mathbf{n}_{i}, \mathbf{p}\right)}{2 \Delta r_{i}} \\
\Delta_{i}^{p} f & =\frac{f\left(\mathbf{r}, \mathbf{p}+\mathbf{m}_{i}\right)-f\left(\mathbf{r}, \mathbf{p}-\mathbf{m}_{i}\right)}{2 \Delta p_{i}}
\end{aligned}
$$

$\mathbf{v}(\mathbf{p})$ and $\mathbf{F}(\mathbf{r})$ are respectively the velocity and the force. In the last equations $\mathbf{n}_{i}$ and $\mathbf{m}_{i}$ are the vectors connecting the centers of close neighbour cells in the direction $i$. The 
non-zero components of these lattice vectors are the previously introduced lattice cells size $\Delta r_{i}$ and $\Delta p_{i}$ respectively. It is easily verified that the particles number and the total energy are conserved also in this discretized form of the Vlasov equation.

The GE can be then defined as

$$
\begin{aligned}
S= & \frac{-g}{N_{h}} \sum_{\mathbf{r}, \mathbf{p}}\left[f^{\prime}(\mathbf{r}, \mathbf{p}) \ln f^{\prime}(\mathbf{r}, \mathbf{p})+\right. \\
& \left.\left(1-f^{\prime}(\mathbf{r}, \mathbf{p})\right) \ln \left(1-f^{\prime}(\mathbf{r}, \mathbf{p})\right)\right]
\end{aligned}
$$

where $f^{\prime}=f h^{2}, g$ is the spin-isospin degeneracy factor ( $g=4$ in our case) and $N_{h}=h^{2} / \Delta \Omega$ is the number of cells contained inside a volume of size $h^{2}, \Delta \Omega$ being the cell volume (this normalization ensures that the number of quantal states is correctly counted). This definition goes into the usual (coarse grained) thermodynamical entropy in the case of thermodynamical equilibrium.

The variation of entropy, according to the finite difference equation of motion (3), is given by

$$
\begin{array}{r}
\frac{\partial S}{\partial t}=\frac{\Delta \Omega g}{N_{h}} \sum_{\mathbf{r}, \mathbf{p}} \sum_{i j} \\
{\left[\frac{v_{i}}{2 \Delta r_{i}} f^{\prime}\left(\mathbf{r}+\mathbf{n}_{i}, \mathbf{p}\right) \ln \frac{f^{\prime}(\mathbf{r}, \mathbf{p})}{f^{\prime}\left(\mathbf{r}+\mathbf{n}_{i}, \mathbf{p}\right)}+\right.} \\
\frac{F_{j}}{\Delta p_{j}} f^{\prime}\left(\mathbf{r}, \mathbf{p}+\mathbf{m}_{j}\right) \ln \frac{f^{\prime}(\mathbf{r}, \mathbf{p})}{f^{\prime}\left(\mathbf{r}, \mathbf{p}+\mathbf{m}_{j}\right)}+ \\
\frac{v_{i}}{2 \Delta r_{i}}\left(1-f^{\prime}\left(\mathbf{r}+\mathbf{n}_{i}, \mathbf{p}\right)\right) \ln \frac{\left(1-f^{\prime}(\mathbf{r}, \mathbf{p})\right)}{\left(1-f^{\prime}\left(\mathbf{r}+\mathbf{n}_{i}, \mathbf{p}\right)\right)}+ \\
\left.\frac{F_{j}}{\Delta p_{j}}\left(1-f^{\prime}\left(\mathbf{r}, \mathbf{p}+\mathbf{m}_{j}\right)\right) \ln \frac{\left(1-f^{\prime}(\mathbf{r}, \mathbf{p})\right)}{\left(1-f^{\prime}\left(\mathbf{r}, \mathbf{p}+\mathbf{m}_{j}\right)\right)}\right]
\end{array}
$$

which is different from zero whenever the distribution $f$ is not uniform and $\Delta \Omega \neq 0$. In particular spontaneous symmetry breaking can occur as in the case of chaotic dynamics. Notice that $\frac{\partial S}{\partial t}=0$, if $f^{\prime}=1 / 2$, as it happens e.g. at the Fermi energy for a Fermi distribution. This is exactly what happens when the linear response is applied to a uniform system at a given temperature. We will discuss later this important point.

Similarly we define the corresponding GE per particle $\sigma$ as

$$
\sigma=\frac{S}{\sum_{\mathbf{r}, \mathbf{p}} f(\mathbf{r}, \mathbf{p})}=\frac{S}{A}
$$


being A the total particle number.

It has to be noticed that in the usual continuous Boltzmann equation the source of entropy is the collision term, which is treated within the assumption of molecular chaos. This assumption, as it is well known, is an ad hoc hypothesis, which introduces irreversibility in the otherwise reversible evolution of the classical system. Here we are exploring a complementary source of irreversibility, namely the filling of phase space due to the strong chaoticity of the time evolution of the system at the mean field level. Notice, in fact, that the entropy of eq.(6) is a single particle entropy, while, in general, one should consider a $N$-body entropy, for which the distribution in the full $2 \mathrm{~N}$-dimensional phase space should be involved. Anyhow, the addition of the collision term could further increase the GE production rate also of the single particle entropy. It should be noticed that the GE for the Landau-Vlasov equation has already been used in heavy-ion collisions, see for example refs. [14].

\section{MULTIFRAGMENTATION: RESULTS AND DISCUSSION}

\section{A. Entropy production: exact numerical results}

In order to investigate the process of fragment formation, we analyzed the behavior of nuclear matter in the spinodal zone of the phase diagram, where uniform matter is unstable with respect to density fluctuations. For this purpose we chose the average nuclear matter initial density smaller than $2 / 3 \rho_{0}$ and the temperature $T=3 \mathrm{MeV}$, i.e. nuclear matter is prepared well inside the unstable region. Since in our simulations we neglected the dynamical evolution which drives the system inside the spinodal zone, we imposed a very small and uniform white noise on the initial average density profile. This random initialization will mimic the initial missing dynamics, perturbing the unstable equilibrium and forcing the system to relax towards a more stable configuration. Within this scheme, the time evolution of a typical single trajectory, as well as the one of a bunch of trajectories, has been extensively analyzed in literature [18 20]. In particular, the appearance of deterministic chaos after an 
initial linear evolution plays a crucial role in the process of fragment formation [19,20]. The details of the interaction could slightly modify the evolution time as found in ref. [21], but not the character of the dynamics.

With reference to the discussion in the previous section, we have to stress that, with the above mentioned initial conditions inside the spinodal region, the equilibrium configuration corresponds to non-homogeneous matter, since fragments are spontaneously formed during the evolution due to the instability of the system. The equilibrium configuration should correspond to a well defined distribution of fragments in thermal equilibrium among each other and with a vapour, but, due to the finite size of the system, large fluctuations in the fragment distribution occur from one event to another. For this reason we do not consider the whole set of possible outcomes all together, but we prefer to initialize the system according to the above mentioned prescriptions, which correspond to include and average over a subset of MF processes for each event (i.e. a single computer run). This sub-set is specified by the initial distribution in phase space as described by the occupation probability $f(\mathbf{r}, \mathbf{p})$ which specifies the average number of particles $f \Delta \Omega$ in each phase space cell and therefore gives a classical coarse grained description of the initial condition.

A typical single event is shown in fig.2, where we plot the time evolution of the density $\bar{\rho}(x)$. The initial average density is taken equal to $0.55 \rho_{0}$. We notice that the small random noise on the initially uniform density profile (please notice the different scale used in the top panel of fig.2) is rapidly amplified by the action of the effective one-body field, thus leading towards fragment formation. An analysis of the Fourier spectra of the excited modes has shown a strong coupling with a sensitive dependence on the initial conditions [20].

Following the definition of the previous section we show in fig.3 (panels (a) and (c)) the time variation of the fraction of occupied phase space cells $N / N_{\text {tot }}$ for MF events started at initial density $\bar{\rho} / \rho_{0}=0.55$ and the corresponding coarse-grained GE's (panels (b) and (d)). In the figure we display the simulation for different widths of the gaussian $\mu$ used to smooth the density in the x-direction and different sizes of the cells. In panels (a) and (c) we observe an increase of the fraction of occupied phase space cells $N / N_{\text {tot }}$ at the time 
corresponding to the fragment formation, being the latter dependent on the gaussian width $\mu$ [21], i.e. on the range of the interaction and on the size of the grid. More precisely we get a slower evolution when using a larger $\mu$ [21] and a greater increase when increasing the size of the cell. We notice that the filling of phase space shows the same time evolution of the GE, which is shown in panels (b) and (d). Therefore MF is strongly characterized by a substantial increase in the GE which reflects, as in the example of the standard map previously discussed, the dynamical filling of phase space due to chaotic motion. The GE is a powerful tool to measure the volume of the occupied phase space, though in a relative way due to the grid dependence. All the calculations that will be shown in the following have been performed using $\mu=0.61 \mathrm{fm}$ and $\Delta x=0.3333 \mathrm{fm}$.

It can be very instructive to calculate the different contributions to the entropy $\sigma$ coming from the surface and the volume of the formed fragments. For this we have to define exactly surface and volume of the two-dimensional fragments formed during the dynamical evolution.

Let us define the surface of the fragments as the ensemble of coordinate space cells where the density $\bar{\rho}(x)$ takes values between the limits $\tilde{\rho}_{1}$ e $\tilde{\rho}_{2}$ defined by

$$
\begin{aligned}
& \tilde{\rho}_{1}=\frac{1}{10} \rho_{\max } \\
& \tilde{\rho}_{2}=\frac{9}{10} \rho_{\max }
\end{aligned}
$$

being $\rho_{\max }$ the highest value of the density $\bar{\rho}(x)$.

Analogously we define the bulk of the fragments as the ensemble of coordinate space cells where the density $\bar{\rho}(x, y)$ is larger than $\tilde{\rho}_{2}$ of eq.(10). For completeness we also characterize the gas component as the ensemble of space cells where the density is smaller than $\tilde{\rho}_{1}$ of eq.(9). Briefly we can summarize by writing

$$
\begin{array}{cl}
\tilde{\rho}_{1}<\bar{\rho}(x)<\tilde{\rho}_{2} \quad & \text { surface } \\
\bar{\rho}(x) \geq \tilde{\rho}_{2} \quad \text { bulk }
\end{array}
$$




$$
\bar{\rho}(x) \leq \tilde{\rho}_{1} \quad \text { gas }
$$

We have checked that this particular choice of the upper and lower density limits produces numerically robust results.

Once the fragment has been defined, we can easily calculate the relative contributions to the entropy per particle $\sigma$ coming from the surface and the bulk of the fragments, and from the gas. For this purpose we denote with $S_{i}$ the GE of the component $i$ and $A_{i}$ the corresponding number of particles. We define the entropy per particle of the component $i$ by

$$
\sigma_{i}=\frac{S_{i}}{A_{i}}
$$

It can be easily checked that the total entropy per particle $\sigma$ can be expressed as a weighted sum of $\sigma_{i}$ over all components, i.e.

$$
\sigma=\frac{S}{A}=\frac{1}{A} \sum_{i} S_{i}=\sum_{i}\left(\frac{A_{i}}{A}\right) \sigma_{i}
$$

Therefore each component contributes to $\sigma$ with the following amounts

$$
\begin{gathered}
\sigma_{\text {surf }}=\frac{-g}{A_{\text {surf }} N_{h}} \sum_{\tilde{\rho}_{1}<\bar{\rho}(x)<\tilde{\rho}_{2}}\left[f^{\prime} \ln f^{\prime}+\left(1-f^{\prime}\right) \ln \left(1-f^{\prime}\right)\right] \\
\sigma_{\text {bulk }}=\frac{-g}{A_{\text {bulk }} N_{h}} \sum_{\bar{\rho}(x) \geq \tilde{\rho}_{2}}\left[f^{\prime} \ln f^{\prime}+\left(1-f^{\prime}\right) \ln \left(1-f^{\prime}\right)\right] \\
\sigma_{\text {gas }}=\frac{-g}{A_{\text {gas }} N_{h}} \sum_{\bar{\rho}(x) \leq \tilde{\rho}_{1}}\left[f^{\prime} \ln f^{\prime}+\left(1-f^{\prime}\right) \ln \left(1-f^{\prime}\right)\right]
\end{gathered}
$$

The onset of MF can be characterized by the time variation of the fraction $\alpha_{i}$ of coordinate space cells occupied by the gas, the bulk and the surface 2 . For them the following relation holds

\footnotetext{
${ }^{2}$ The reader should notice that $\alpha$ is defined in "coordinate space" and is different from the previously defined $N / N_{t o t}$, being the latter the number of occupied "phase space" cells.
} 


$$
\alpha_{\text {surf }}+\alpha_{b u l k}+\alpha_{\text {gas }}=1
$$

This is clearly shown in fig.4, panels (a) and (c). In particular we display in panel (a) the time variation of the fraction of space cells belonging to the bulk (circles) and the surface (dashed line), whereas in panel (c) we show the variation of the fraction of space cells belonging to the gas (dotted line). Those results concern a system initialized at density $\bar{\rho}=0.55 \rho_{0}$. We clearly observe their sudden change at the time when fragments form. In particular, the gaseous and the surface parts are zero before fragments form, whereas the bulk fraction is dominant. During fragment formation, their relative contribution change, the bulk contribution decreases while the gas and the surface grow until fragments are completely formed. At this time, which we define as the fragmentation time $\tau_{\text {frag }}$, all the $\alpha^{\prime} s$ reach a plateau, apart from some small fluctuations. We have checked that this behavior is quite general and does not depend on the initial density, as can be seen in panels (a) and (c) of fig.5, where the same results are plotted for an initial density $\bar{\rho}=0.3 \rho_{0}$.

At the fragmentation time an appreciable increase of the entropy per particle $\sigma$ is observed, see fig.4(b). There we display the time variation of the total entropy per particle $\sigma$ (solid line), the bulk (circles) and the surface entropy (dashed line) for a system initially prepared at an average density $\bar{\rho}=0.55 \rho_{0}$. At fragmentation time $\tau_{\text {frag }} \simeq 160 \mathrm{fm} / \mathrm{c}$ the increase of the entropy per particle is about $\Delta \sigma \sim 0.4$ and is independent on the initial average density which, on the other hand, determines the fragmentation time. This is clearly shown in panel (b) of fig.5, where the case for a system with initial density $\bar{\rho}=0.3 \rho_{0}$ is displayed. Here the fragmentation time is shorter than the previous case, $\tau_{\text {frag }} \simeq 80 \mathrm{fm} / \mathrm{c}$.

Please note also the relative contributions of the surface and the bulk. The surface entropy (dashed line) shows a strong increase from zero to 0.9 . When the surface forms, the surface entropy is practically equal to the total entropy per particle, $\sigma_{\text {surf }} \sim \sigma$. We have checked that this relation actually holds for all initial average densities, see fig.5(b). Therefore the main mechanism of entropy production appears to be the formation of surfaces region, which in the final stage include a substantial fraction of the total number of particles. 
On the contrary, the bulk entropy $\sigma_{b u l k}$ (circles in panel (b) of figs.4 and 5) does not change appreciably during the time of fragmentation and keeps close to the initial entropy per particle $\sigma$.

As far as the gas is concerned, we notice that the process of fragmentation is followed by the formation of a very excited and rarefied gas. As one can see from figs. 4(d) and $5(\mathrm{~d})$, the gas entropy per particle $\sigma_{\text {gas }}$ takes values about a factor of three bigger than the surface and bulk entropies, although large fluctuations show up during the fragmentation. The gas component is, however, quite small and gives an almost negligible contribution to the total entropy $S$ and to the total entropy per particle $\sigma$. Evidences of a larger entropy per particle of the gas component has been found in the analysis of experimental data [14], and the values we find in our simulations seem to be typical for MF reactions [14,8]. The gas temperature has been found to be very high and will be discussed in the following 8 .

\section{B. Entropy production: exact numerical results vs. linear response}

In this subsection we compare the exact numerical evolution with the one obtained using linear response [22] starting from the same initial condition. In agreement with ref. [21], we find that in the initial stage of the time evolution the linear response is a good approximation to the exact one. In the case reported in fig. 6 for the initial density $\rho=0.4 \rho_{0}$ (this initial density has been chosen in order to facilitate the comparison with ref. [21]) we see that up to 40-50 fm/c the two profiles are similar, but they differ drastically as time goes on. Moreover the linear response violates strongly energy and number of particles conservation after $70-80 \mathrm{fm} / \mathrm{c}$. At that time the density profile becomes also negative. But apart from this considerations which, though qualitatively similar, depend on the initial density and the folding used for the response, the most important difference between the two time evolutions is that linear response does not produce any growth of GE at variance with the exact result (see fig.6(f)). This fact is easily understood considering what already noticed at the end of section II. The variation of entropy with time is zero if the occupation number $f^{\prime}$ changes 
around $1 / 2$, i.e. very close to the Fermi energy. This is what happens for the linear response which is not able to thermalize the system. Therefore this finding confirms the crucial role of nonlinearity and chaos, giving at the same time a strong support to the conjecture advanced in ref. [31] for chaotic maps. Unfortunately it is very difficult in our case to calculate all the Lyapunov spectrum and verify that the slope of the linear growth of the GE gives the KS entropy.

\section{Final temperature of the fragments}

Since the average density rapidly changes from the low initial values to almost the saturation during the same interval of time, we conclude that the temperature of the fragments increases with respect to the initial value $(\mathrm{T}=3 \mathrm{MeV})$. This is indeed the case, as it is shown in fig.7(a). There we plot the one-body distribution function, averaged over a large number of cells in coordinate space, as a function of the energy. The histogram indicate the results of the numerical calculation (one typical event), whereas the solid line is a fit with a FermiDirac distribution function at temperature $T=7 \mathrm{MeV}$. This finding is weakly dependent on the initial average density, as clearly shown in fig.8(a), where the final fragment temperature is slightly higher $(\mathrm{T}=8 \mathrm{MeV})$ and the system is initialized at density $\bar{\rho} / \rho_{0}=0.3$. Some details on the study of the distribution function are given in Appendix.

In figs.7(b) and 8(b) we display a typical distribution function of the gas vs. the energy density, averaged over a finite number of cells. From this distribution, which does not resemble a maxwellian because of the high momenta tails, we can extract the temperature of the gas (see Appendix) and this turns out to be much larger than the fragments' temperature. More precisely we get for the initial density $\bar{\rho} / \rho_{0}=0.55 \quad T_{\text {gas }}=30.4 \mathrm{MeV}$, while $T_{\text {gas }}=$ 15.8 MeV for $\bar{\rho} / \rho_{0}=0.3$. Therefore the system formed by fragments plus gas is on the whole not equilibrated, being the two components at very different final temperatures. Though these numbers are not the average over many simulations, this result is typical, i.e. repeating the calculation one gets a similar result. 
This fact is consistent with some features observed in the experimental data. In ref. [6] it has been found that the apparent temperatures extracted from the slopes of the spectra of the emitted light particles $\left(\mathrm{p}, \mathrm{d},{ }^{3} \mathrm{He}\right)$ are in general greater than those measured by other experimental groups [3,5] through the ratio of the double isotope yields [32]. In ref. [5] it has been claimed that such particles are emitted in a first stage of the collision and not from a source in thermal equilibrium at variance with the intermediate mass fragments (IMF). Then, notwithstanding the simplifications of our numerical simulations, we find close analogies with the experimental data. At the moment, the fact that we obtain a gas with a temperature higher by a factor of two-three cannot allow definite conclusions. The comparison with the experimental data is more qualitative than quantitative. However, both theory and experiments seem to strongly indicate that the temperatures extracted from the light particles might not carry information on the thermal equilibrated source of MF. Thus the apparent temperatures extracted from the slopes may be misleading for extracting the nuclear caloric curve and one should be very careful in using them.

Finally, in fig.9 we draw the 2D equation of state (EOS) for nuclear matter with the same Skyrme forces employed for this model, see refs. [20] for more details. The solid line is the isothermal curve at temperature $\mathrm{T}=0$, the dashed line at $\mathrm{T}=3 \mathrm{MeV}$ and the patterned area represents the region where our fragments lie after the dynamical evolution started in the spinodal region. The circle (square) represents the final state of the fragments when the system is started at $\bar{\rho} / \rho_{0}=0.3(0.55)$. Error bars indicate uncertainties on their final density. We see that the final formed fragments are stable and close to thermodynamical equilibrium. This plot confirms that the numerical simulation is fully consistent and reliable. From this result and from the moments of the mass distributions reported in ref. [20], we can argue that while the events corresponding to an initial density 0.55 are very close to the MF phase transition point (power law in the mass distribution), those corresponding to 0.3 correspond to a fragment production of smaller size and therefore to a higher initial excitation energy. The value we get for the temperature of the fragments in the two cases $(7$ and $8 \mathrm{MeV}$ respectively) therefore indicate a rise in the caloric curve of the kind observed 
by the Aladin and the Indra groups [3:6]. Again the comparison is more qualitative than quantitative.

\section{CONCLUSIONS}

Within the framework of the Vlasov equation solved in a 2D lattice by means of a deterministic algorithm we have studied the process of fragment formation in MF events. The model has already been used with success in the past to clarify the dynamics of nuclear matter inside the spinodal region. In the present paper we have studied the time evolution of the coarse-grained GE when MF occurs. It has to be stressed that two-body collisions are missing in our model. Despite that, we have found that the GE increases rapidly at the moment of fragment formation and saturates soon after. This behavior confirms the

role of chaos in filling dynamically the available phase space ( in the coarse-grained sense ), as measured by the GE increase. In fact, only if the dynamics is chaotic, and therefore mixing, the initial smooth distribution is able to spread in phase space until the reaching of equilibrium. In this process the initial distribution is expected to change shape and form a rather irregular pattern with a sharp increase of the size of its boundary. This means that the momentum distribution will be strongly dependent on the position. Consequently the density will tend to vary from one place to another, which favours fragment formation. This picture is consistent with the fact that the main source of entropy turns out to be the surface regions of the system. These results are at variance with those obtained using the linear response which is not able to thermalize the system and does not give any growth of entropy.

The general trend of the entropy values are actually not far from the ones discussed in the literature in connections with the analysis of several experimental data [14.8]. Of course, due to the schematic character of the model, no detailed comparison with experiments is possible.

We have found a relaxation to equilibrium for what concerns intermediate mass fragments 
(IMF). The density of the final fragments is fully consistent with the calculated EOS. On the other hand we find a gas which is not in equilibrium with the liquid part (IMF), having a temperature which is a factor of 2-3 higher. Though the role of the collision term should be better investigated in this respect, this feature has been found also experimentally and could be very general. Finally the temperature of the fragments corresponding to higher excitation energy is slightly larger. The latter is a very preliminary result which will be investigated with more detail in the future.

\section{ACKNOWLEDGMENTS}

We thank V. Latora for helpful and stimulating discussions. Clarifying discussions with M. Colonna are also acknowledged.

\section{APPENDIX A:}

In order to investigate equilibrium properties of nuclear matter we studied the momentum distribution function $f_{b u l k}(p)$

$$
f_{\text {bulk }}(p)=\frac{\int f\left(x, y, p_{x}, p_{y}\right) \delta\left(x-x_{F}\right) \delta\left(p-P^{1 / 2}\right) d \Gamma}{\int \delta\left(x-x_{F}\right) \delta\left(p-P^{1 / 2}\right) d \Gamma}
$$

being $P=p_{x}^{2}+p_{y}^{2}, f\left(x, y, p_{x}, p_{y}\right)$ is the occupation probability, $d \Gamma$ is the phase space volume element and $x_{F}$ is an ensemble of cells in coordinate space, inside a given fragment, over which the distribution function is averaged.

In order to calculate the fragment temperature we have minimized the function

$$
\chi^{2}(\mu, T)=\frac{\int\left|f_{\text {bulk }}(\epsilon)-f^{F D}(\epsilon, \mu, T)\right|^{2} d \epsilon}{\int d \epsilon}
$$

being $\epsilon=p^{2} / 2 m$ and $f^{F D}(\epsilon, \mu, T)$ is the Fermi-Dirac distribution

$$
f^{F D}(\epsilon, \mu, T)=\frac{1}{1+\exp ((\epsilon-\mu) / T)}
$$

keeping $\mu$ e $T$ as free parameters to be fitted. 
We proceed in a similar way in order to investigate the equilibrium properties of the gas $(\bar{\rho} \leq 0.05)$. For this purpose we have studied the distribution function $f_{\text {gas }}(p)$

$$
f_{\text {gas }}(p)=\frac{\int f\left(x, y, p_{x}, p_{y}\right) \delta\left(x-x_{g}\right) \delta\left(p-P^{1 / 2}\right) d \Gamma}{\int \delta\left(x-x_{g}\right) \delta\left(p-P^{1 / 2}\right) d \Gamma}
$$

being $f$ the occupation probability and $d \Gamma$ the phase space volume element. $x_{g}$ is an ensemble of gas cells in coordinate space over which the distribution function is averaged. Therefore we have calculated the effective temperature of the gas as

$$
T_{e f f}=\frac{\int d \Gamma \frac{p^{2}}{2 m} f_{\text {gas }}(p)}{\int d \Gamma f_{\text {gas }}(p)}
$$

In fact considering that we are in 2D and in classical mechanics - due to the high temperatures - the average of the kinetic energy is equal to the temperature. In the case of the discretized version of the Vlasov equation, instead of the integrations and of the $\delta$-functions, the appropriate summations and discrete $\delta$ 's must be used.

(i) E-mail: atalmi@ct.infn.it

(ii) E-mail: baldo@ct.infn.it

(iii) E-mail: burgio@ct.infn.it

(iv) E-mail: rapisarda@ct.infn.it 


\section{REFERENCES}

[1] J.E. Finn et al., Phys. Rev. Lett. 75, 1321 (1982).

[2] G. Bertsch and J.P. Siemens, Phys. Lett. 126B, 9 (1983); J.P. Siemens, Nature (London) 305, 410 (1983).

[3] J. Pochodzalla et al. Phys. Rev. Lett. 49, 1040 (1995).

[4] M.L. Gilkes et al., Phys. Rev. Lett. 73, 1590 (1994).

[5] J.A. Hauger et al., Phys. Rev. Lett. 77, 235 (1996).

[6] Y.G. Ma et al. Phys. Lett. B 390, 41 (1997).

[7] P.F. Mastinu et al., Phys. Rev. Lett. 76, 2646 (1996).

[8] C. Kuhn et al., FOPI collaboration,Phys. Rev. C48, 1232 (1993) and refs. therein.

[9] For an up-to-date review see for example: Proceed. of the Intern. Conf. CRIS 96, Eds. S. Costa, S. Albergo, A. Insolia and C. Tuvé, World scientific 1996, and refs. therein.

[10] J. Bondorf et al., Nucl. Phys. A 443, 321 (1985); J. Bondorf et al. Phys. Rep. 257, 133 (1995) and refs. therein.

[11] D.H.E. Gross et al., Nucl. Phys. A 443, 321 (1985); D.H.E. Gross et al., Phys. Rep. 279, 119 (1997) and refs. therein.

[12] P. Schuck, R. W. Hasse, J. Jaenicke, C. Gregoire, B. Remaud, F. Sebille and E. Suraud, Prog. Part. Nucl. Phys. 22, 181 (1989); W. Cassing, V. Metag, U. Mosel and K. Niita, Phys. Rep. 188, 363 (1990).

[13] G.F. Bertsch and S. Das Gupta,Phys. Rep. 160, 189 (1988).

[14] L.P. Csernai and J. Kapusta, Phys. Rep. 131, 223 (1986) and refs. therein.

[15] X. Campi, J. Phys. A19, L917 (1986); Phys. Lett. B208, 351 (1988). 
[16] W. Bauer, Phys. Rev. C38, 1297 (1988);

[17] V. Latora, M. Belkacem and A. Bonasera, Phys. Rev. Lett. 73, 1765 (1994); P. Finocchiaro et al., Nucl. Phys. A572, 477 (1996).

[18] G.F. Burgio, Ph. Chomaz and J. Randrup, Phys. Rev. Lett. 69, 885 (1992); G.F. Burgio, Ph. Chomaz, M. Colonna and J. Randrup, Nucl. Phys. A581, 356 (1995).

[19] G.F. Burgio, M. Baldo and A. Rapisarda, Phys. Lett. B321, 307 (1994); M. Baldo, G.F. Burgio and A. Rapisarda, Nucl. Phys. A583, 343 (1995).

[20] M. Baldo, G.F. Burgio and A. Rapisarda, Phys. Rev. C51, 198 (1995). A. Atalmi, M. Baldo, G.F. Burgio and A. Rapisarda, Proceedings of the XXXIV Winter Meeting on Nuclear Physics, 22-27 January, 1996, Bormio, Italy; A. Atalmi, M. Baldo, G.F. Burgio and A. Rapisarda, Phys. Rev. C53, 2556 (1996); A. Atalmi, M. Baldo, G.F. Burgio and A. Rapisarda, in ref. [9].

[21] B. Jacquot, M. Colonna, Ph. Chomaz and A. Guarnera, Phys. Lett. B359, 268 (1995); B. Jacquot et al., Phys. Rev. C54, 3025 (1997).

[22] M. Colonna, Ph. Chomaz, J. Randrup, Nucl. Phys. A567, 637 (1994).

[23] A. Bonasera, V. Latora and A. Rapisarda Phys. Rev. Lett. 75, 3434 (1995).

[24] L. Boltzmann, Ann. Phys. 57, 773 (1896); J. L. Lebowitz, Phys. Today, 32, September (1993).

[25] S.K. Nayak, R.Ramaswamy and C. Chakravarty, Phys. Rev. E51, 3376 (1995).

[26] Ch. Dellago, H.A. Posch and W.G. Hoover, Phys. Rev. E 531485 (1996); Ch. Dellago and H.A. Posch, Physica A230, 364 (1996).

[27] L. Caiani, L. Casetti, C. Clementi and M. Pettini, Phys. Rev. Lett. 79, 4361 (1997). L. Caiani, L. Casetti, C. Clementi, G. Pettini, M. Pettini and R. Gatto,Phys. Rev. E57, 3886 (1998). 
[28] V. Latora, A. Rapisarda and S. Ruffo, Phys. Rev. Lett. 80, 692 (1998) and Physica D (1998) in press, chao-dyn/9803019; M. Antoni and S. Ruffo, Phys. Rev. E52, 2361 (1995).

[29] V.I. Arnold and A. Avez, Ergodic problems of Classical Mechanics, New York Benjamin (1968).

[30] H.G. Schuster, Deterministic chaos, VCH (1995).

[31] V. Latora and M. Baranger, MIT-CTP preprint no. 2751, chao-dyn/9806006.

[32] S. Albergo et al., Il Nuovo Cimento 89A, 1 (1985).

[33] K. Huang, Statistical Mechanics, J. Wiley and Sons (1963).

[34] O. Penrose, Rep. Prog. Phys. 42, 129 (1979). 


\section{FIGURES}

FIG. 1. Filling of phase space for the standard map (top panels) for two values of $\mathrm{K}=0.05,2$ and respective time evolution of the coarse-grained GE. The slope of the linear rise of S gives the KS entropy, i.e. the largest Lyapunov exponent in this case [31].

FIG. 2. For a typical event, we plot the space density $\bar{\rho}(x)$ at different times. The initial average density $\bar{\rho}$ is equal to $0.55 \rho_{0}$. Please notice the different scale used in the top panel.

FIG. 3. A typical time evolution of the occupation number is shown in panels (a) and (c) for different choices of cell size $\Delta x$ and the gaussian width $\mu$. In panels (b) and (d) the total entropy per particle is shown. The solid lines represent calculations performed with $\Delta x=0.66 \mathrm{fm}$, whereas the dashed line the ones at $\Delta x=0.33 \mathrm{fm}$. See text for details.

FIG. 4. The time evolution of $\alpha$ (the number of coordinate space cells) and $\sigma$ are shown in panels (a) and (b) for the bulk (circles) and the surface (dashed line). The gas components, $\alpha_{\text {gas }}$ and $\sigma_{\text {gas }}$ (dotted line) are shown in panels (c) and (d) for a typical trajectory at an initial density $\bar{\rho} / \rho_{0}=0.55$. The solid line in panels (b) and (d) represents the total entropy per particle. Arrows indicate the fragmentation time $\tau_{\text {frag. }}$.

FIG. 5. Same as Fig.4, but for a typical trajectory at an initial density $\bar{\rho} / \rho_{0}=0.3$. 
FIG. 6. The exact numerical evolution (full line) and the linear response one (dashed line) are compared for the density profiles (panels (a)-(e)) and for the entropy evolution (panel (f)). The linear response is able to follow the exact evolution approximately only up to $50 \mathrm{fm} / \mathrm{c}$. For later times, the difference between the two profile increases and the evolution of the linear response becomes unreliable: the violation of energy and particles number is substantial already at $60 \mathrm{fm} / \mathrm{c}$, while at $80 \mathrm{fm} / \mathrm{c}$ the profile becomes also negative. However the entropy production is the real big difference between the two approaches. As shown in panel (f) the linear response does not produce any entropy growth at variance with the exact simulation. See text for further details.

FIG. 7. In panel (a) we plot (histogram) a typical one-body distribution function $f(\epsilon), \epsilon=P^{2} / 2 m$, calculated in the bulk of the fragments (and averaged over all fragments) at the final time $\mathrm{t}=200 \mathrm{fm} / \mathrm{c}$. The initial average density is $\bar{\rho} / \rho_{0}=0.55$. The solid line represents the fit performed with a Fermi-Dirac distribution with a temperature $T$ (see Appendix). We get an average fragment temperature $\mathrm{T}=7 \mathrm{MeV}$. In panel (b) we show the distribution function for the gas. In this case the calculation of the temperature as discussed in the Appendix gives $\mathrm{T}=30.4 \mathrm{MeV}$.

FIG. 8. Same as Fig.7 for $\bar{\rho} / \rho_{0}=0.3$ at the final time $\mathrm{t}=100 \mathrm{fm} / \mathrm{c}$.

FIG. 9. The pressure is plotted vs. the density for a 2D system with the same Skyrme forces used for the model investigated (see ref. 201). The solid line represents the EOS at zero temperature, the dashed line the one at $\mathrm{T}=3 \mathrm{MeV}$, whereas the hatched area encloses the EOS's between $\mathrm{T}=6.5 \mathrm{MeV}$ and $\mathrm{T}=8.5 \mathrm{MeV}$. The filled circle represent the average final state of the fragments when the nuclear system is initialized at $\bar{\rho} / \rho_{0}=0.3$, whereas the filled square concerns the one at $\bar{\rho} / \rho_{0}=0.55$. The error bars indicate uncertainties on their final density. 
Fig.1 Atalmi, Baldo, Burgio and Rapisarda
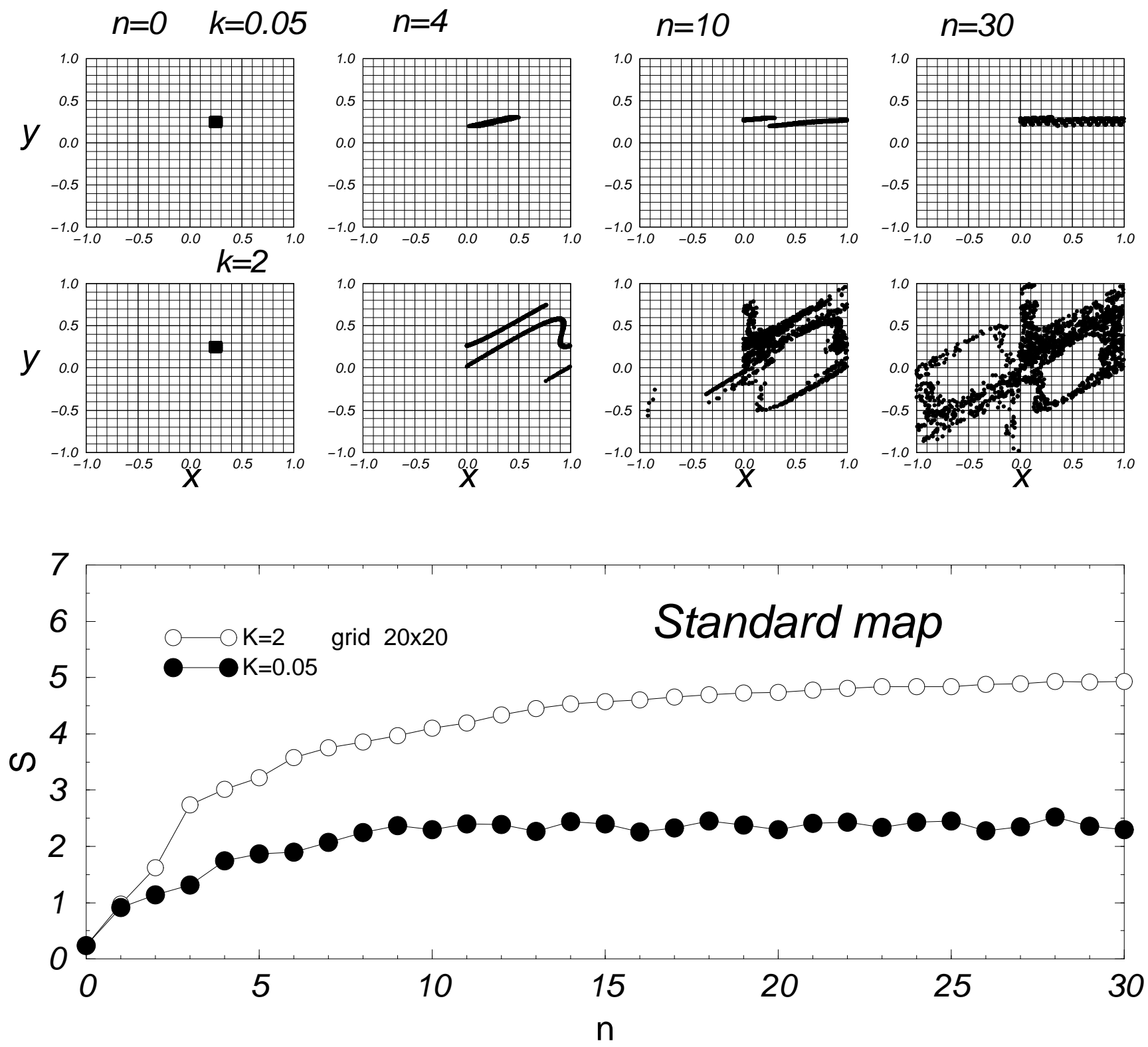
Fig.2 Atalmi, Baldo, Burgio and Rapisarda

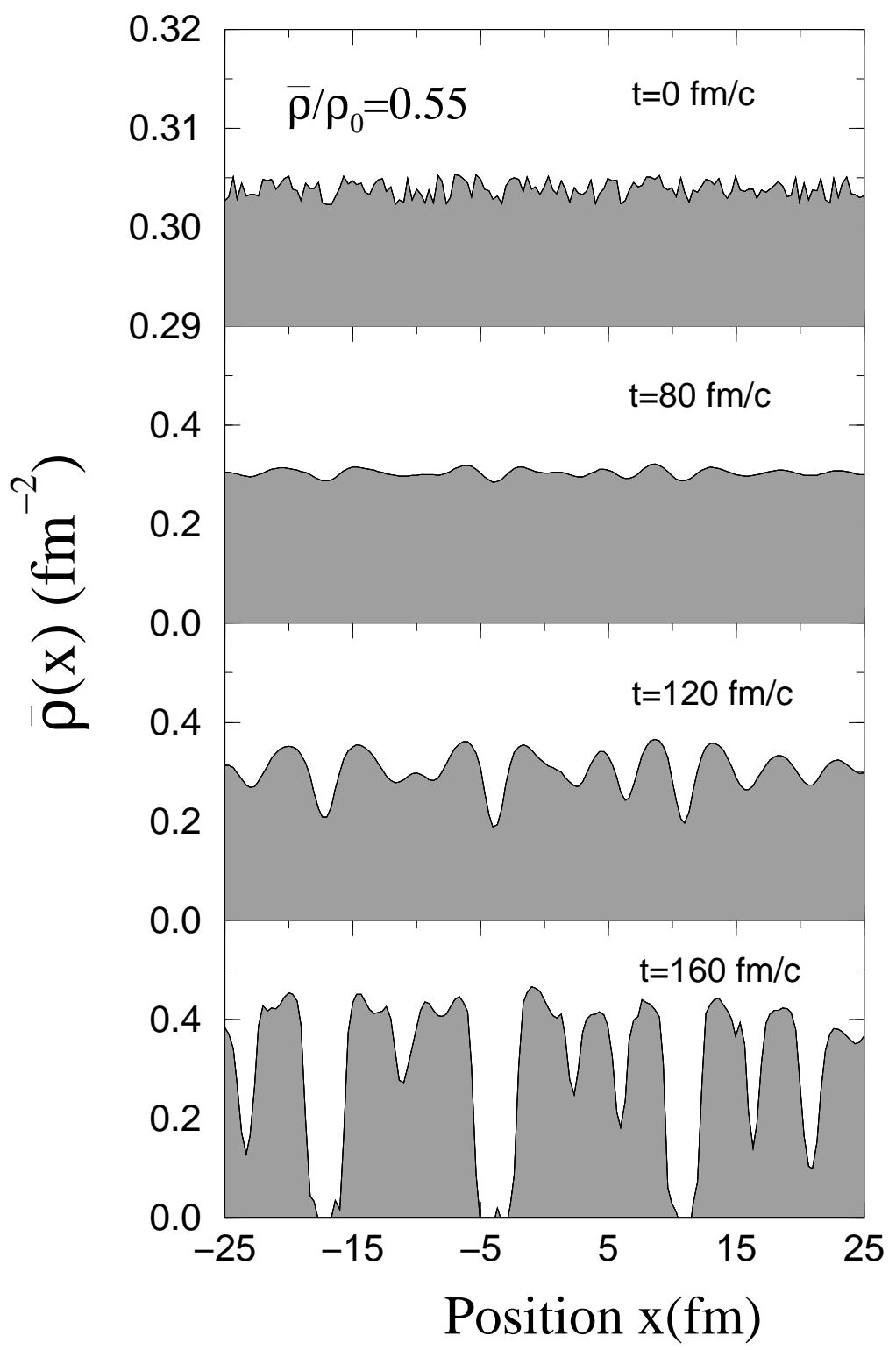


Fig.3 Atalmi, Baldo, Burgio and Rapisarda
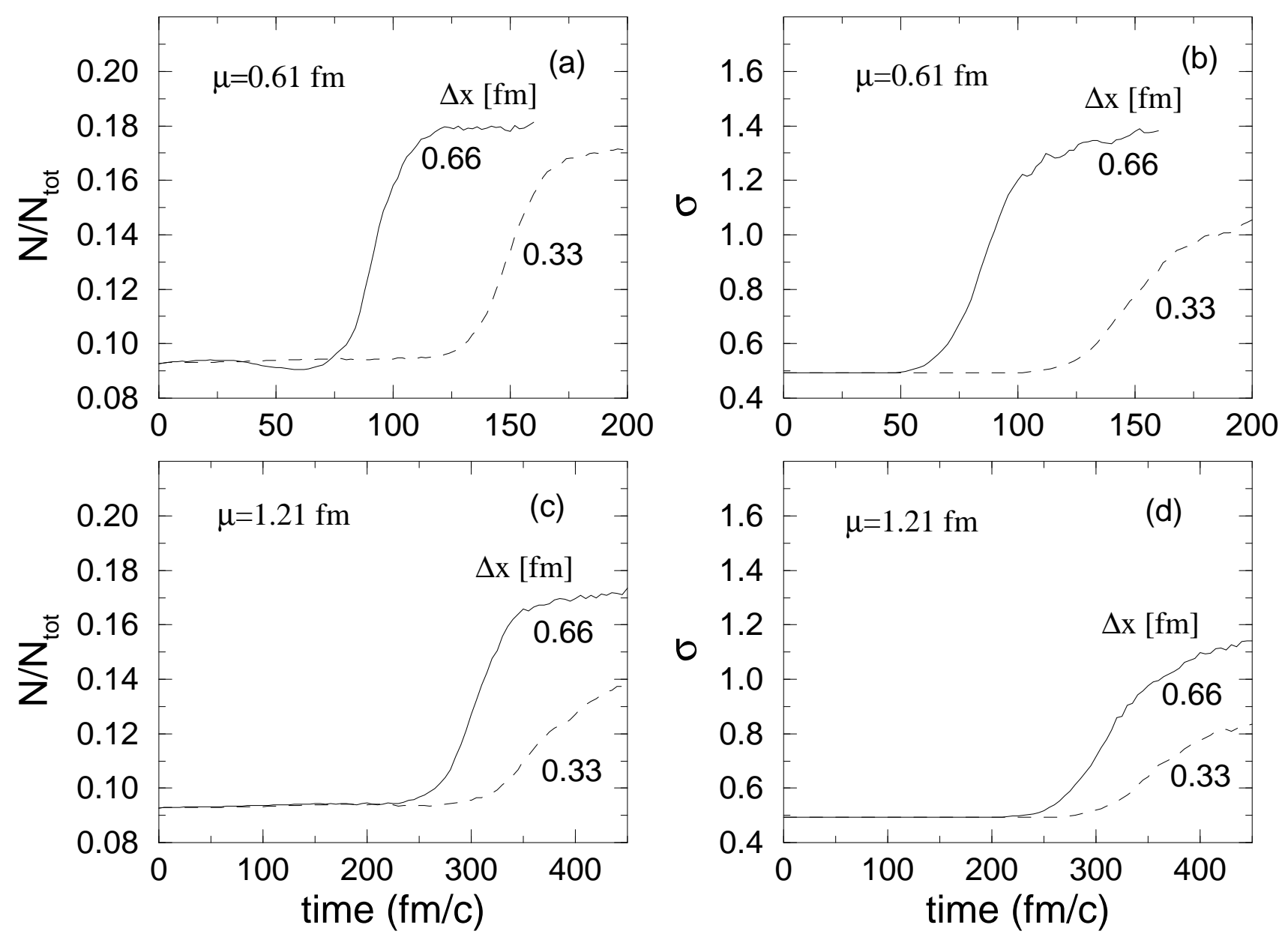

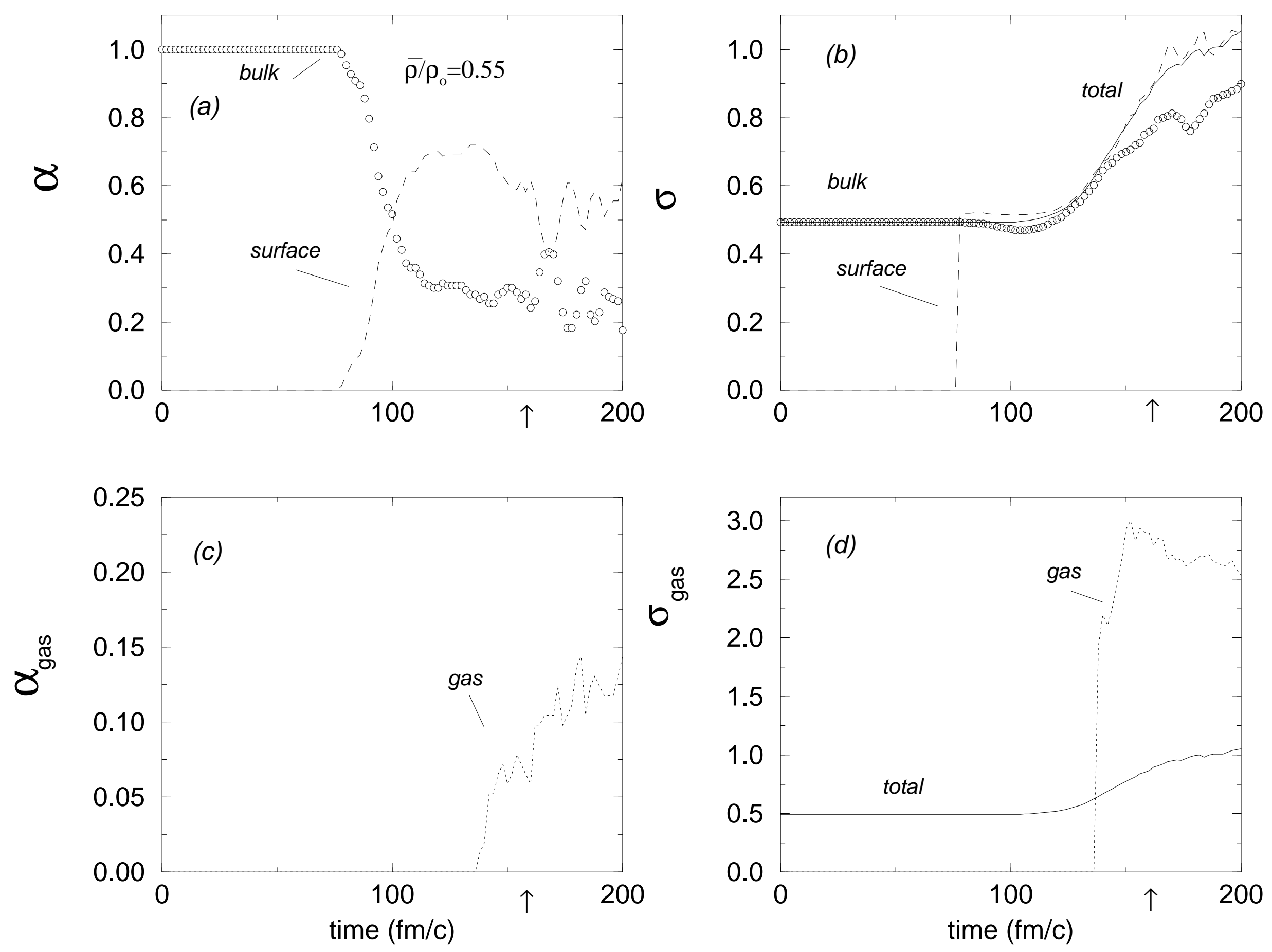

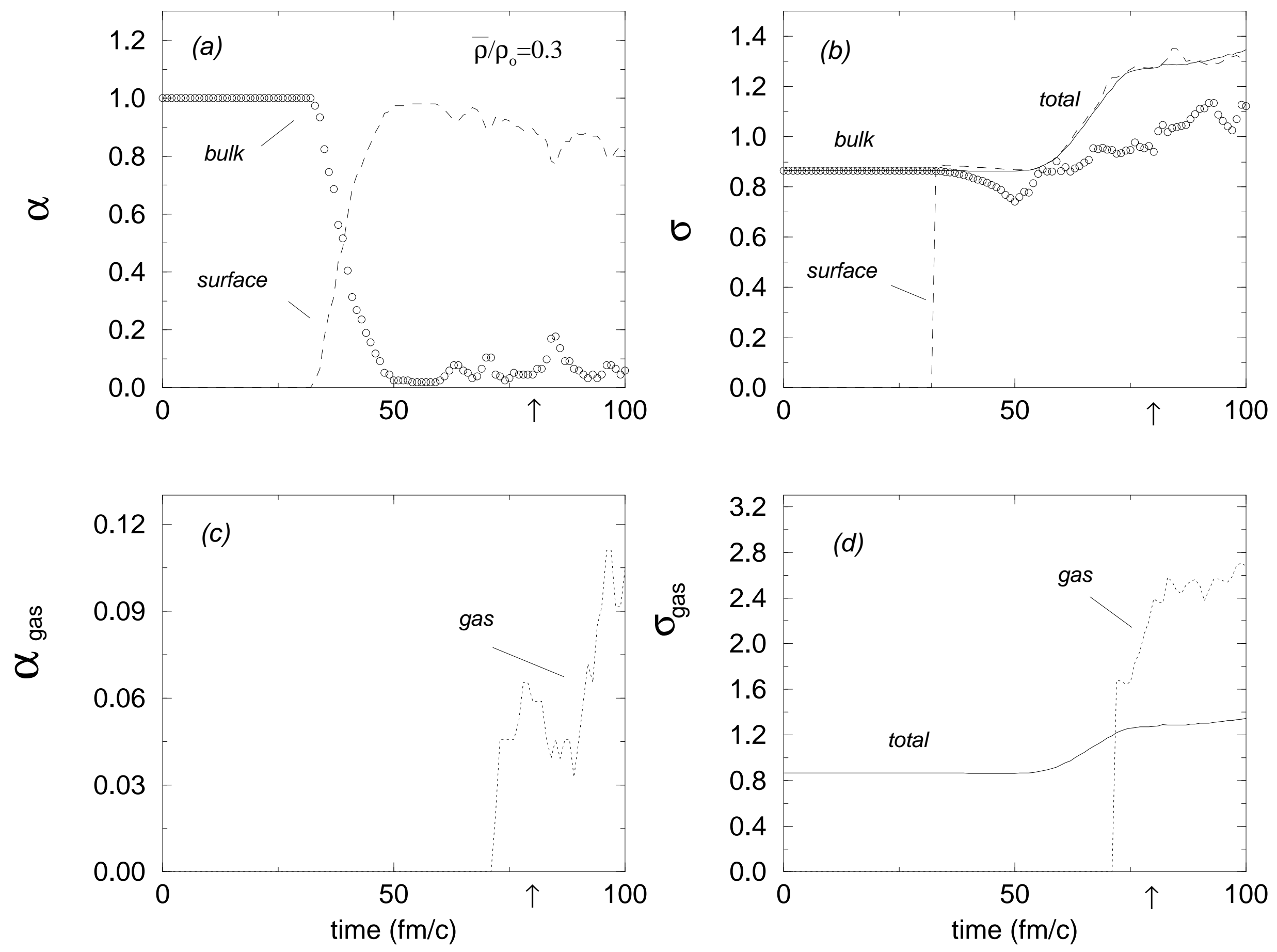
Fig.6 (new) Atalmi, Baldo, Burgio and Rapisarda

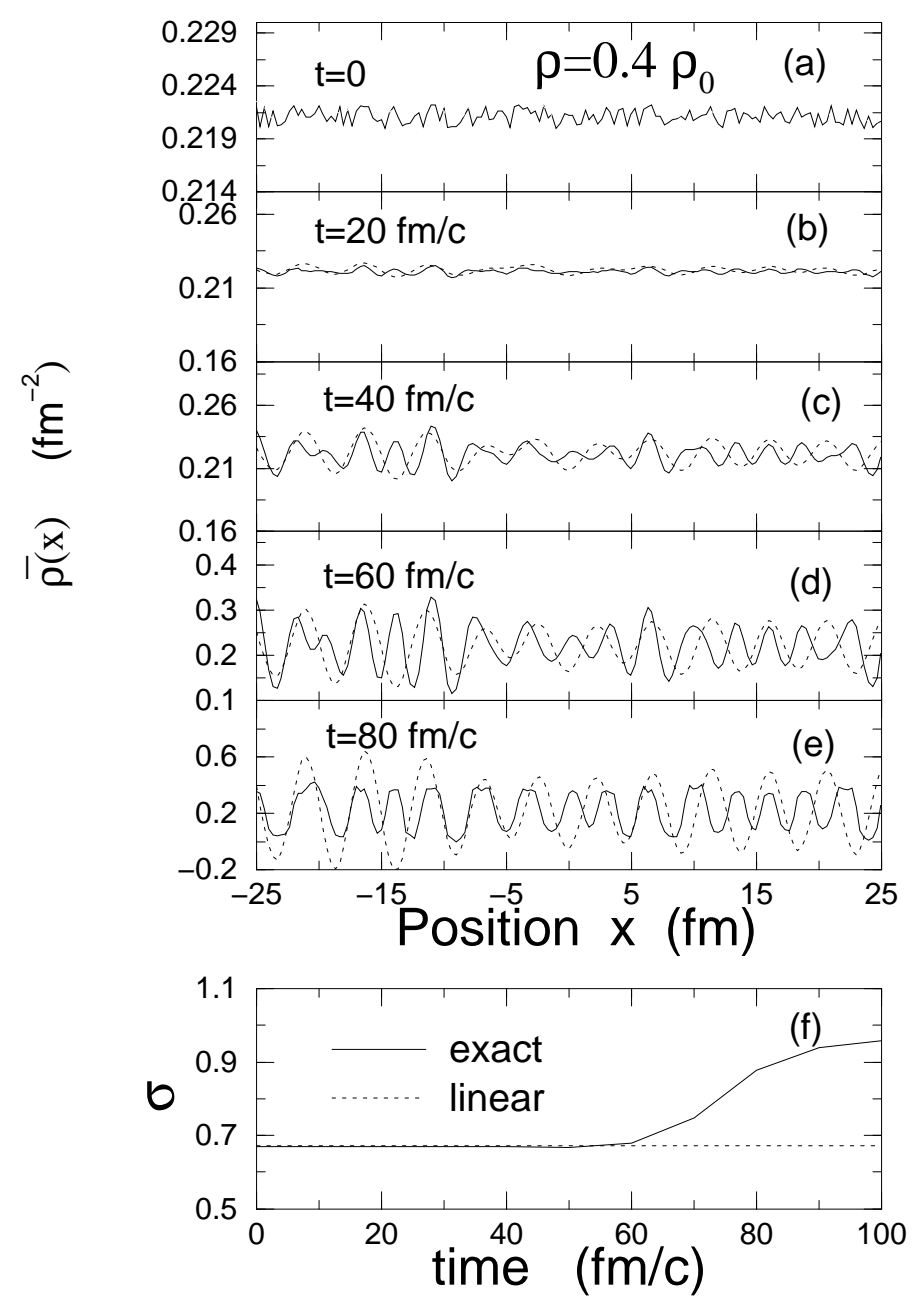


Fig.7 Atalmi, Baldo, Burgio and Rapisarda (revised)
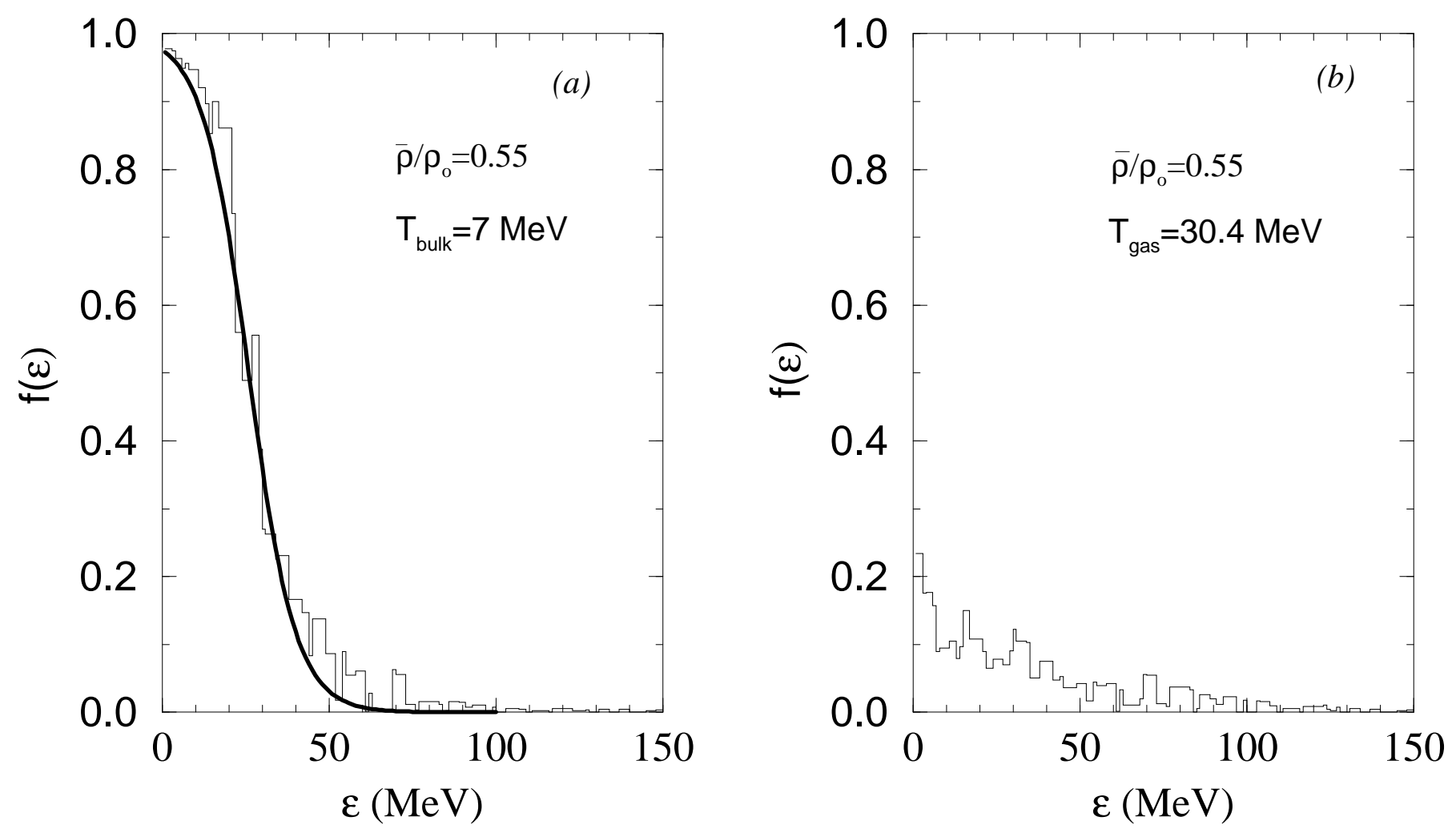
Fig.8 Atalmi, Baldo, Burgio and Rapisarda (revised)
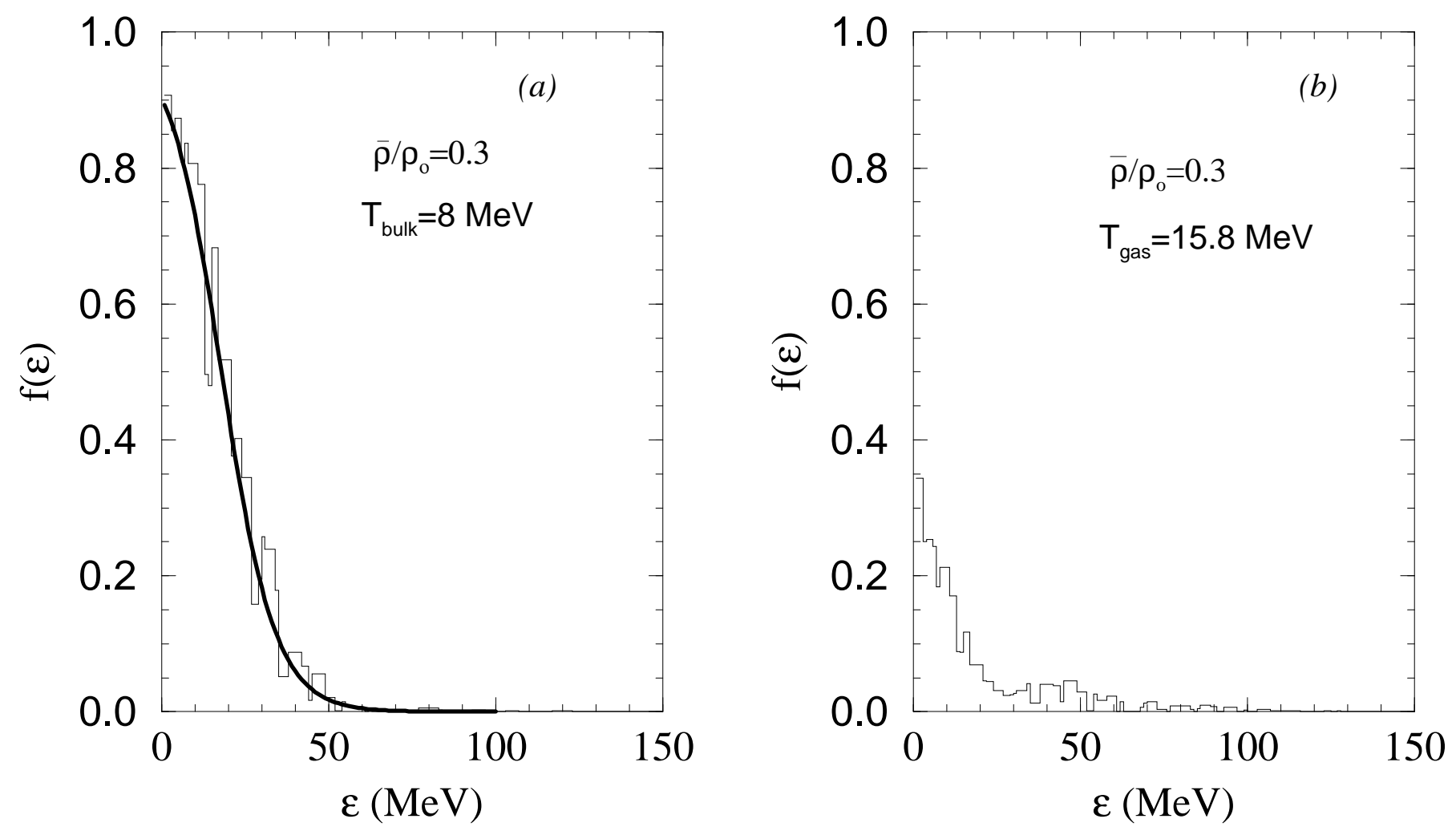
Fig.9 Atalmi, Baldo, Burgio and Rapisarda

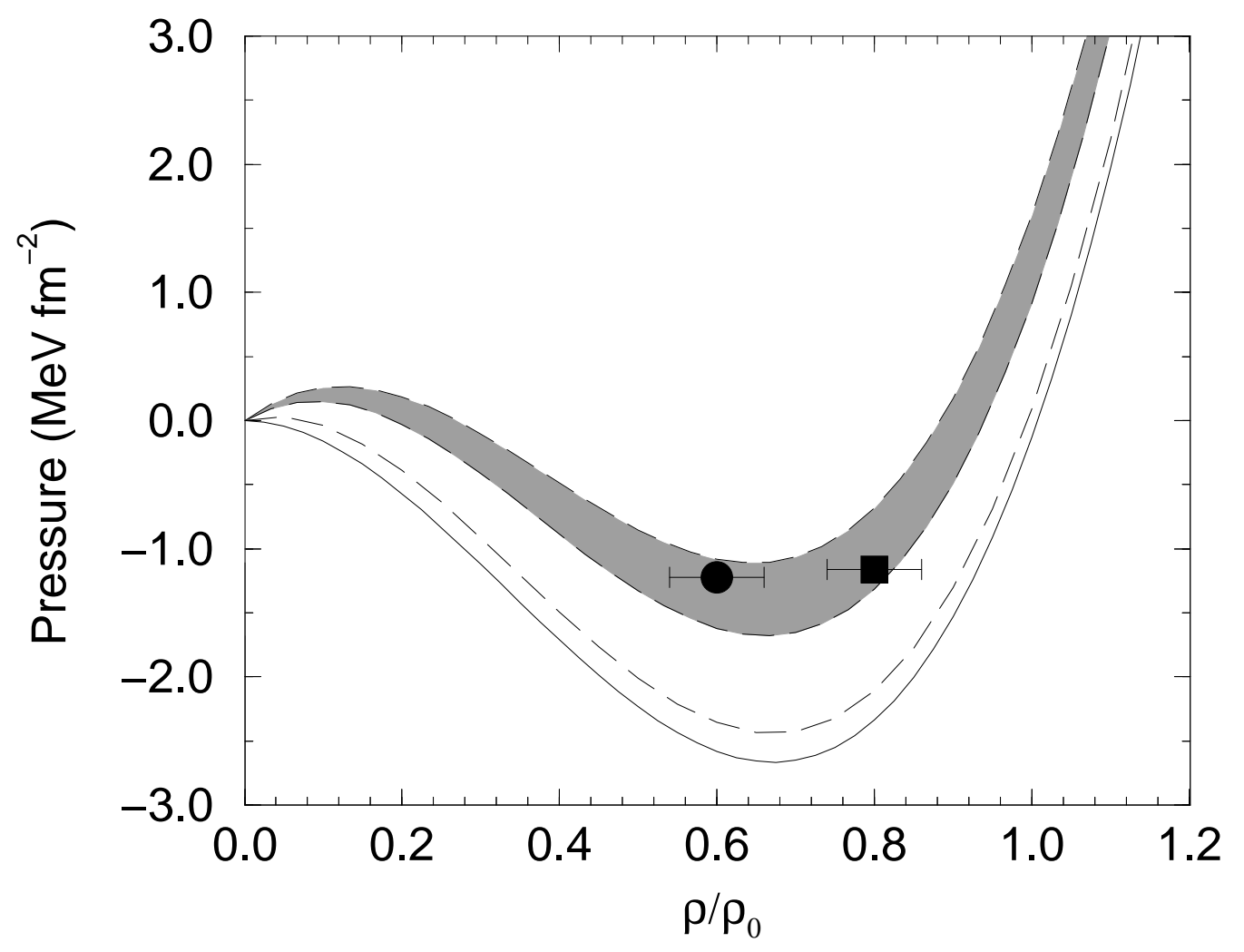

